\title{
Optimization of Building Components with Sustainability Aspects in BIM Environment
}

\author{
Mostafa Khanzadi', Ali Kaveh*, Mohammad Rastegar Moghaddam', \\ Seyyed Mohammad Pourbagheri²
}

\author{
Department of Civil Engineering, Iran University of Science and Technology \\ Narmak, Tehran, P.O. Box 16846-13114, Iran \\ 2 Department of Computer Engineering, Iran University of Science and Technology \\ Narmak, Tehran, P.O. Box 16846-13114, Iran \\ * Corresponding author, e-mail: alikaveh@iust.ac.ir
}

Received: 19 May 2018, Accepted: 10 October 2018, Published online: 06 November 2018

\begin{abstract}
In recent decades, the variety of building materials has grown a great deal causing the selection of suitable materials from a wide range of candidates to be complex and difficult. One of the main criteria to be considered in this area, besides reducing procurement cost, is paying attention to various aspects affecting the dimensions of sustainable development, such as increasing energy saving, applying recyclable materials and localization.

This paper proposes a framework in the BIM environment - as one of the successful approaches in the AEC industry - which allows the project stakeholders to choose the most desired and optimal combination for their building components with least human interference in the selection process makes systematic choices. In order to achieve the purposes embedded in the framework phases, several methods such as ENSCBO, DEA and VIKOR have been utilized. Afterwards, to evaluate and depict the selection process, this is implemented as a Revit plugin and eventually applied to a case study.
\end{abstract}

Keywords

building components optimization, sustainable development, BIM, ENSCBO, DEA, VIKOR

\section{Introduction}

Ideal construction and urbanism development must be such that reaching our requirements does not prevent future generations from suitable life [1]. No doubt, the construction industry, as one of the major consumers of energy, materials, and land space, has great implications on human environment and achieving sustainable development in construction industry has become an inevitable necessity.

Utilizing appropriate materials for each individual building component is one of the most important factors which distinguish durable buildings from low life and it is completely in line with sustainable development.

In the past, the range of building materials were limited, but in the recent decades, traditional materials are being replaced by the new ones, in order to satisfying various needs such as performance improvement [2]. This deployment makes the process of selecting the materials in appropriate way, difficult, and struck between several alternatives in order to meet design objectives and criteria.
This is a complex decision-making process in which many preconditions, requirements, and considerations are involved [3]. The ultimate goal of this process is to select materials such that certain criteria are optimized. Some of these criteria or objectives are cost, energy, environmental impacts etc. Complexity of multi-objective problems is that optimizing one objective will not necessarily lead to the optimization of others, and a trade-off between multiple objectives is required [4]. With these explanations, it is necessary to follow a pre-defined framework in order to select materials in the early design processes and pre-construction phases.

Nowadays, extensive application of computers and engineering software, demonstrate their tremendous role in Architecture, Engineering and Construction (AEC) industry. It is known that the Building Information Modeling (BIM) is a data-rich, intelligent, and parametric digital representation of objects [5] which considers 
the entire life cycle of the project in design and documentation [6]. Also, BIM as a vital approach for extracting and transferring data between the project participants, and consequently, analyzing and making their decisions, could be prominent tool [7]. Thus, collaboration between computers and engineering software, BIM approach, optimization algorithms, multi-criteria decision analysis techniques and other methods can help decision makers.

Many researches have been attracted in the field of material selection. As mentioned, this problem is generally known as a multi criteria decision making (MCDM) problem, some papers considered this problem as multi attributes decision making (MADM) and others view this problem as multi objective decision making (MODM). In the following, some such studies are investigated.

Ashby [4] solved this problem by combining multiple performance metrics into one as a value function. This is done by defining exchange constants for each metrics. Then he solved this single-objective optimization problem by different approaches.

Another approach based on Grey relational analysis and end-of-life product strategy is presented by Chan and Tong [8] that performs this decision-making process by ranking the materials with respect to different criteria and uses multi-criteria weighted average for this. Considered requirements in this paper do not only include technical and economic factors, but also the environmental factors.

Rao [2] used an improved compromise ranking method for evaluating several materials with respect to measures of the quantitative and qualitative attributes and relative importance of each criterion in an engineering application.

Usually the products are bundled by the suppliers in order to increase the orders of their company. Wu et al. [9] considered this problem, known as "bundling problem", to select among notebook suppliers in Taiwan. They used the criteria gathered from experts and Analytic Network Process (ANP) to build a model, and then from the results of the ANP model, coefficients of the objective function in Mixed Integer Programming (MIP) were determined to address the bundling problem.

Zhou et al. [10] considered environmental impacts in addition to technical and economic factors. Then this multi-objective optimization problem was solved by Artificial Neural Networks (ANN) combined with Genetic Algorithm (GA).

The environmental impacts are also considered by Castro-Lacouture et al. [11] and rating was performed using Leadership in Energy and Environmental Design
(LEED) for green performance of building projects and the corresponding multi-objective problem was solved by mixed integer optimization method.

Florez et al. [3] developed a framework for green material selection by considering the impact of sustainability perceptions. In other words, the subjective judgment of consumer about a product was considered in addition to the objective factors such as design and cost considerations. The final objective of this framework was the maximization of the gathered points based on material-related LEED-based credits.

Hamdy et al. [12] used Genetic Algorithm as multi-objective optimization approach and combined it with IDA ICE (building performance simulation program) and proposed a method for minimizing the carbon dioxide equivalent emissions and the investment cost. They considered heating/cooling energy source in addition to heat recovery type, and used six building envelope parameters in their study as design variables.

Ogunkah and Yang [5] identified some factors that could affect on architects in their decision-making process in their choice of green + building materials. They used Analytic Hierarchy Process (AHP) technique for evaluating and selecting the building material.

Akadiri et al. [6] employed the Fuzzy Extended Analytical Hierarchy Process (FEAHP) technique to develop a model for selecting sustainable materials in building projects. They considered twenty-four criteria for sustainable material selection and reduced them into six assessment factors. The perceived importance of these criteria was determined using questionnaire and then the numerical values were determined using FEAHP.

Florez and Castro-Lacouture [13] proposed a model based on mixed integer optimization and considered subjective factors in addition to objective factors. They used a factor analysis approach to measure the subjective factors.

Marzouk et al. [14] proposed a framework that integrates Building Information Modeling (BIM), Life Cycle Cost (LCC) and Saudi Arabia Green Buildings Rating System (SAGRS). Also they used Genetic Algorithm (GA) for optimization purposes in their framework.

Jalaei et al. [15] integrated Decision Support System (DSS), BIM and LCC in order to use the potential of BIM to assess the impacts of various alternatives of materials on energy saving in the building's life cycle. They used the Technique for Order of Preference by Similarity to Ideal Solution (TOPSIS) method to develop DSS and select the optimum type of building components and design families. 
Integrating BIM and LCC in their work moves the design decisions forward at the early stage in conceptual design phase of project. They also used numerical models for simulating alternative situations and ranking them based on owners' priorities and sustainability criteria.

Liu et al. [16] also utilized BIM environment to optimize the buildings' design and to improve their sustainability. The optimization method used in their work was Particle Swarm Optimization (PSO) by which they applied trade-off between LCC and Life Cycle Carbon Emissions (LCCE) for different designs. In this method, continuous and discrete factors were considered simultaneously and as a result, the size of searching space will have been increased. They applied their method on an office building in Hong Kong for evaluating it and the results showed its reliability, effectiveness and efficiency.

Another use of BIM in material selection was presented by Akanmu et al. in [17]. They combined a modified harmony search optimization algorithm and supplier performance rating in developing their decision support system. The modified harmony search they used includes supplier selection criteria and relevant rating. The obtained results showed the good capability of this model in material selection and overall improvement of building design process.

Govindan et al. [18] proposed a hybrid multi-criteria decision making process that uses DANP for analyzing the impacts of criteria on each other, and TOPSIS for identifying the best alternative with respect to those criteria. They first selected suitable sustainable indicators for the best construction material, then revealed the most important and influential indicator among them, and finally found the best material by their proposed method.

Dawood [19] proposed a method using the BIM approach as a simulation tool and GA as an optimization algorithm to find near-optimal solution. The data and information needed were obtained by BIM and the parametric programming language (created by DYNAMO plug in) was used to facilitate interoperability between BIM and other parts of the model.

Ahmad et al. [20] used BIM to develop an economic sustainability assessment framework for residential buildings and used LCC as a traditional indicator and some non-traditional indicator (affordability, manageability and adaptability) in their framework. They also used objective and subjective indicators in their work.

In this study, an almost automated framework is developed for choosing best options available for each building components. The main feature of this framework is that it minimizes the user intervention as much as possible and makes systematic choices. In subsequent section, the phases of the framework are completely described. In Section 3, the utilized algorithms and methods are thoroughly explained. Implementation of this by developing a plugin and applying to a case study are presented in Section 4. Finally, in the last section concluding remarks are provided.

\section{Proposed framework for selection of desired and optimum building components}

Presently, with the profound help and impact of employing BIM technology in AEC industry and its consequences on raising productivity, manufacturers of the building's elements provide their products with BIM files (families). These files include materials and mechanical properties, energy analysis, suppliers' data and a lot of practical data that can be added, available on online libraries such as SmartBim ${ }^{\mathrm{TM}}$ platform. Therefore, project stakeholders can apply and evaluate these pre-made family files in their conceptual design stage and ultimately choose the best option according to their judgment.

The proposed framework of this research for opting desired and optimum selection for building components with considering sustainable aspects in BIM environments consists of four sequential phases: "Initial preparation", "optimization", "efficiency evaluation" and "multi-attributes decision making". All these phases are integrated and implemented by developing a plug-in within BIM tool (e.g., Autodesk Revit). These phases and related methods are described in the following. Architecture of this framework is illustrated in Fig. 1.

\subsection{Initial preparation phase}

At this phase, the 3D model of the selected project with sufficient corresponding details is created in a BIM environment such as Revit software in order to satisfy the required Level of Development (LOD). Then the desired elements that owner or designer wants to be participated in the optimization process, including doors, windows, walls, ceilings, etc. are selected. Also, at this phase, an external database of predefined candidates for each element is created by designer. Members of this database can be recognized by BIM tool (with the format of either RFA or RVT format). This database supports optimization phase and provides its requirements.

\subsection{Optimization phase}

Since there are many possible combinations of components to be selected in the previous phase, evaluation of all combinations cannot be considered. Thus to opt the optimal 


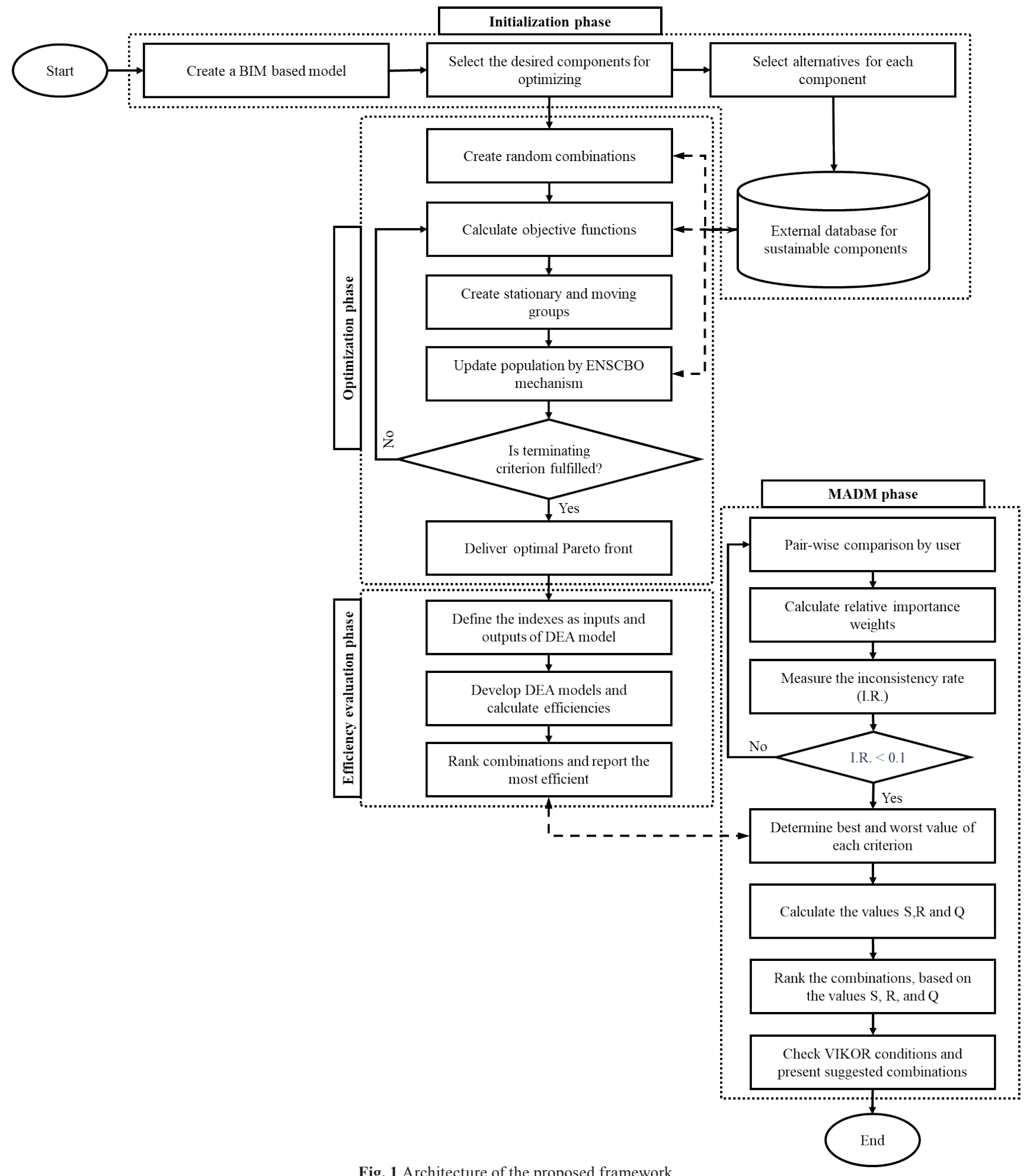

Fig. 1 Architecture of the proposed framework

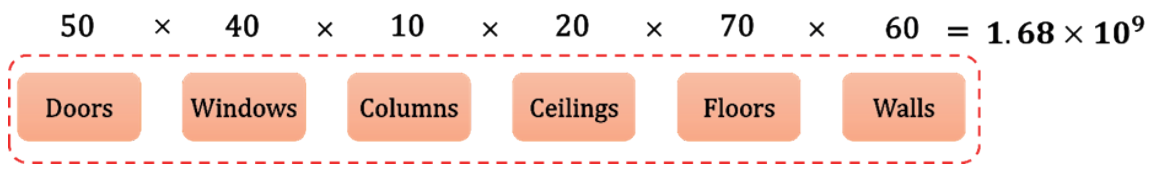

Fig. 2 An example for possible number of combinations 
combination of components, a meta-heuristic algorithm can be utilized. In Fig. 2, possible number of combinations for a hypothetical example is illustrated.

In this phase, based on the concept of population based algorithms, a random combination of building components is generated first. The size of this initial population is much smaller than the number of possible combinations. At the next level, this population is evaluated according to the various objective functions. These objectives are trying to meet the various dimensions of sustainable development.

After calculating the objective functions for each member of random population, desirability of them is found and sorted. At the next step, this combination should be updated to reach a better situation. Updating procedure depends on the employed meta-heuristic algorithm. In this study Enhanced Non-Dominated Sorting Colliding Bodies Optimization (ENSCBO) is selected. As it is known there is not a unique optimal answer in multi-objective problems. Instead, a set of answers is said to be the final result and called optimal Pareto front. Each member of final Pareto front has certain superiority over the rest, so none of them is the best. This final Pareto front feeds the next phase.

\subsection{Efficiency evaluation phase}

Although the optimization phase restricts the span of the combination selection and just delivers the non-dominated ones; however various combinations are still eligible to be selected, so they must be filtered again. In this framework, this is done by calculating the efficiency of each proposed combination with the help of Data Envelopment Analysis (DEA) method. The efficiency of each combination is equal to the ratio of the weighted sum of incremental objectives (such as recyclable materials) to the weighted sum of subtractive objectives (such as total components' cost). After calculating the efficiency of each elements combination, the most efficient combinations qualify and the rest of them are eliminated from the selection process.

\subsection{Multi-attributes decision making phase}

After computing the efficiency of optimal Pareto front members, the best ones enter to this phase. To select one combination of components as final decision, multi-attributes decision making (MADM) methods are required. In these methods, final choice is determined based on the user and owners' strategic approach. In order to calculate the importance weights of each criterion, a pair wise comparison is made between each two criteria, and after checking inconsistency rate, these final weights are calculated. Finally, the efficient alternatives are ranked according to the chosen MADM methods' procedure. In this paper VIKOR is utilized according to its successes.

\section{Methods used in the proposed framework}

Metaheuristic algorithm are well developed in the last three decades, some recent metaheuristics are presented in the book by Kaveh [21], and other efficient methods can be found in the work of Refs. [22-25].

In order to achieve the purposes embedded in the considered phases, several techniques are employed in this framework. In following these techniques are explained in detail.

\subsection{Enhanced Non-Dominated Sorting Colliding Bodies Optimization (ENSCBO)}

In optimization phase, we have used Enhanced NonDominated Sorting Colliding Bodies Optimization (ENSCBO) algorithm as a multi-objective version of recently developed meta-heuristic algorithm, Enhanced Colliding Bodies Optimization (ECBO). The ECBO algorithm is a modified version of the standard Colliding Bodies Optimization (CBO) algorithm.

The CBO is a population-based meta-heuristic algorithm, which is developed by Kaveh and Mahdavi [26]. This algorithm is originally inspired from nature, specifically from one-dimensional collisions between bodies. Collisions between bodies occur based on two laws of physics; the laws of conservation of momentum and energy. These two laws are conserved in an isolated system, while the collision is taking place [27]. This algorithm, by imitating this phenomenon, provides a method for solving complex optimization problems. Additional explanations can be found in Refs. [28, 29].

Kaveh and Ilchi Ghazaan [30] applied two modifications. They improved the standard $\mathrm{CBO}$ in quality of the solutions and convergence speed, and called it ECBO. First modification was using a memory to save the best found solution and the second modification was utilizing a mechanism to escape from local optima.

\subsubsection{The ENSCBO algorithm mechanism}

Like other population based algorithms, the multi-objective version of the ECBO, ENSCBO, developed by Kaveh et al. [27], starts with a population of random colliding bodies (CB). Each CB is known as a solution candidate and has a specific mass. The CBs are evaluated according to the defined objective functions. 
In this stage, CBs are sorted by using non-dominated sorting approach instead of regular sorting according to their fitness values. Non-dominated sorting was utilized in NSGA-II by Deb et al. [31] for the first time. The result of non-dominated sorting is a set of Pareto fronts that satisfy the following expressions:

$n_{p}=\left|\left\{q \in P \mid p \in S_{q}\right\}\right|$

$F_{1}=\left\{p \in P \mid n_{p}=0\right\}$

$F_{i}=\left\{p \in P\left|n_{p}=\right|\left\{q \in F_{j} \mid p \in S_{q} ; \forall j: 1 \leq j \leq i-1\right\} \mid\right\}$

$; \forall i: i \geq 2$

Where $F_{i}$ is the $i$ th Pareto front, $n_{p}$ is the number of solutions which dominate $p, S_{p}$ is the set of solutions that $p$ dominates, and $P$ is the population.

By applying non-dominated sorting on $\mathrm{CBs}$, possible fronts are created and CBs are ranked subsequently. Crowding distance (CD) is another technique which is used for prioritizing $\mathrm{CBs}$ in each front [31]. For each $\mathrm{CB}$ crowding distance is calculated by:

$$
C D^{i}=\sum_{j=1}^{k} \frac{\left|f_{j}^{i+1}-f_{j}^{i-1}\right|}{f_{j}^{\max }-f_{j}^{\min }} \quad j=1, . ., k
$$

Where $f_{i}^{i+1}$ and $f_{i}^{i-1}$ are the $j$ th function value of the $(i+1)^{\text {th }}$ and $(i-1)^{\text {th }} \mathrm{CB}$ in the considered front, respectively. Also $f_{j}^{\max }$ and $f_{j}^{\text {min }}$ are the maximum and minimum values of the $j$ th function, respectively.

By using these two techniques, CBs are fully sorted and divided into two equal groups; i.e. stationary and moving groups. The lower half of the CBs form the stationary group and the upper half contains the moving CBs. The moving CBs move toward stationary CBs and a collision happens between them.

In order to save historical improvement, best $\mathrm{CBs}$ are stored in a memory. This memory is updated in each iteration.

The magnitude of the mass for each $\mathrm{CB}$ is calculated by using the rank number and CD values of the CBs by Eq. (2).

$$
m_{k}=\frac{\frac{1}{\operatorname{Rank}(k)+\frac{1}{C D(k)}}}{\sum_{i=1}^{n} \frac{1}{\operatorname{Rank}(i)+\frac{1}{C D(i)}}}, \quad k=1,2, \ldots, n
$$

The initial velocities of the stationary and moving CBs are specified by:

$$
\begin{aligned}
& v_{i}=0, \quad i=1,2, \ldots, \frac{n}{2} \\
& v_{i}=x_{i-\frac{n}{2}}-x_{i}, \quad i=\frac{n}{2}+1, \frac{n}{2}+2, \ldots, n
\end{aligned}
$$

Where $v_{i}$ and $x_{i}$ are the velocity and location vector of the $i$ th CB in this group, respectively; $x_{i-n / 2}$ is the location of the $i$ th $\mathrm{CB}$ pair.

After the collision, the velocities of the stationary and moving $\mathrm{CBs}$ are updated by:

$$
\begin{aligned}
& v_{i}^{\prime}=\frac{\left(m_{i+\frac{n}{2}}+\varepsilon m_{i+\frac{n}{2}}\right) v_{i+\frac{n}{2}}}{m_{i}+m_{i+\frac{n}{2}}} \quad i=1,2, \ldots, \frac{n}{2} \\
& v_{i}^{\prime}=\frac{\left(m_{i}-\varepsilon m_{i-\frac{n}{2}}\right) v_{i}}{m_{i}+m_{i-\frac{n}{2}}} \quad i=\frac{n}{2}+1, \frac{n}{2}+2, \ldots, n \\
& \varepsilon=1-{\frac{i t e r}{i t e r_{\text {max }}}}
\end{aligned}
$$

Where iter and iter $_{\text {max }}$ are the current iteration number and the total number of iterations for optimization process respectively, and $\varepsilon$ is the coefficient of restitution (COR). Also updated locations of the CBs are calculated as:

$$
\begin{aligned}
& x_{i}^{\text {new }}=x_{i}+\operatorname{rand} \circ v_{i}^{\prime} \quad i=1,2, \ldots, \frac{n}{2} \\
& x_{i}^{\text {new }}=x_{\substack{i-\frac{n}{2} \\
\text { nand }}}+\operatorname{ran} v_{i}^{\prime}, \quad i=\frac{n}{2}+1, \frac{n}{2}+2, \ldots, n
\end{aligned}
$$

Where $x_{i}^{\text {new }}, x_{i}$ and $v_{i}{ }^{\prime}$ are the new location, previous location and the velocity after the collision of the $i$ th $\mathrm{CB}$, respectively. rand is a random vector uniformly distributed in the range of $[-1,1]$.

In order to escape from local optimum, a random number between 0 and $1, r n_{i}(i=1,2,3, \ldots)$, is generated for each $\mathrm{CB}$ and then compared with a parameter like Pro. If $r n_{i}<$ Pro, a random variable $(j)$ of the $i$ th $\mathrm{CB}$ is selected and updated to a random value.

The population updated and evaluated repeatedly to fulfilled termination criterion.

\subsection{Data Envelopment Analysis (DEA)}

Data envelopment analysis (DEA) is a data-oriented approach and a non-parametric programming for efficiency estimation of DMUs (decision-making unit) [32], 
or in the other words, it is a data driven method to conduct relative performance measurements among a set of decision making units. Efficiency is a relative concept of production. The term DMU refers to decision making units with multiple inputs and outputs. In this research each member of final Pareto front is considered as a DMU and objective function values of them known as inputs and outputs. This valuable analytical tool computes the most possible efficiency of each DMU by assigning appropriate weight to inputs and outputs. Charnes et al. [33] developed a simple DEA model, named CCR. This model computes efficiency of each DMU by solving following model:

$$
\begin{array}{ll}
\underset{u, v}{\operatorname{Max}} & \theta=\frac{v^{T} y_{0}}{u^{T} x_{0}} \\
\text { st : } & \\
& v^{T} y_{j}-u^{T} x_{j} \leq 0, \quad j=1,2, \ldots, n \\
& u, v \geq 0
\end{array}
$$

Where $u$ and $v$ are inputs and outputs weights vectors. The $x_{j}$ and $y_{j}$ are inputs and outputs vector of DMUj; $x_{0}$ and $y_{0}$ are inputs and outputs vector of the target $\mathrm{DMU}_{0}$. Finally, $\theta$ represents its efficiency.

After solving this for all DMUs, they are sorted according to their calculated efficiency.

\subsection{The compromise ranking method VIKOR}

The compromise ranking method (VlseKriterijumska Optimizacija I Kompromisno Resenje) VIKOR, is a multi-criteria decision making (MCDM) approach for complex systems optimization in the presence of conflicting and non-commensurable criteria. This method ranks the alternatives and determines the solution named compromise solution due to the particular distance of "closeness" to the "ideal" solution [34]. The VIKOR procedure consists of the following steps:

Step 1: Determine the best, $f_{i}^{*}$, and the worst, $f_{i}^{-}$, values of all criterion functions, $i=1,2, \ldots, n$; where $n$ is the number of criteria.

Step 2: Calculate the values $S_{k}$ and $R_{k}$ by using the Eq. (11) and Eq. (12).

$$
\begin{aligned}
& S_{k}=\sum_{i=1}^{n} w_{i} \frac{\left|f_{i}^{*}-f_{k i}\right|}{\left|f_{i}^{*}-f_{i}^{-}\right|}, k=1,2, \ldots, m \\
& R_{k}=\max \left\{\frac{\left|f_{i}^{*}-f_{k i}\right|}{\left|f_{i}^{*}-f_{i}^{-}\right|}, i=1,2, . ., n\right\}
\end{aligned}
$$

Where $w_{j}$ are the importance criteria weights, obtained in initial. $S_{k}$ expresses the average gap and $R_{k}$ denotes the maximal gap for prioritizing the improvement. Also, $m$ is number of alternatives.

Step 3: Calculate the values $Q_{k}$ by using the Eq. (13).

$Q_{k}=v \frac{\left(S_{k}-S^{*}\right)}{\left(S^{-}-S^{*}\right)}+(1-v) \frac{\left(R_{k}-R^{*}\right)}{\left(R^{-}-R^{*}\right)}$

Where $S^{*}$ and $S^{-}$are minimum and maximum of $S_{k}$ respectively, and $R^{*}$ and $R^{-}$have same definitions. By a moderate strategy, $v=0.5$. Finally, $Q_{k}$ expresses the compromise gap.

Step 4: Rank the alternatives, based on the values $S, R$, and $Q$, in decreasing order in three distinct lists.

Step 5: Alternative $A_{k}$ is compromise solution if it is the best in all three lists. Otherwise, $A_{k}{ }^{\prime}$, best alternative in the $Q$ list, is the compromise solution if it satisfies the following two conditions:

Condition 1: $Q\left(A_{k}^{\prime \prime}\right)-Q\left(A_{k}^{\prime}\right)>\frac{1}{k-1}$, Where $A_{k}{ }^{\prime \prime}$ is the second best in the $Q$ list.

Condition 2: Alternative $A_{k}{ }^{\prime}$ must also be the best ranked in $S$ or $R$ list.

If Condition 1 is satisfied and Condition 2 is not met, $A_{k}{ }^{\prime}$ and $A_{k}{ }^{\prime \prime}$ are solutions. If Condition 1 is not satisfied, alternatives $A_{k}{ }^{\prime}, A_{k}{ }^{\prime \prime}, \ldots, A_{k}{ }^{n}$ are a set of compromised solutions. With the condition that: $Q\left(A_{k}^{\mathrm{n}}\right)-Q\left(A_{k}^{\prime}\right)<\frac{1}{k-1}$.

\section{Implementation on a case study and the corresponding results}

The implementation of the mentioned framework has been accomplished by designing a plug-in in the Autodesk Revit interface. This plug-in enables the extraction of the required data from the BIM model for framework phases. Autodesk Revit provides an Application Program Interface (API) that enables external applications to access it and exchange information. Since API is based on the .NET framework, visual basic.net (VB.net) programming language is used for implementing the plugin. The VB.net is a multi-paradigm programming language with object-oriented and component-oriented features and hence, is appropriate for our design purposes of plugin. The implementation of plugin is done in visual studio 2013.

In order to evaluate the performance of the proposed framework, a residential 32 story high-rise tower, Tavrizh project in Tabriz, which has a total floor area of $36856 \mathrm{~m}^{2}$, is selected as a case study. A rendered snapshot of this project is depicted in Fig. 3. By executing the plugin, system asks to select the desired components and the 


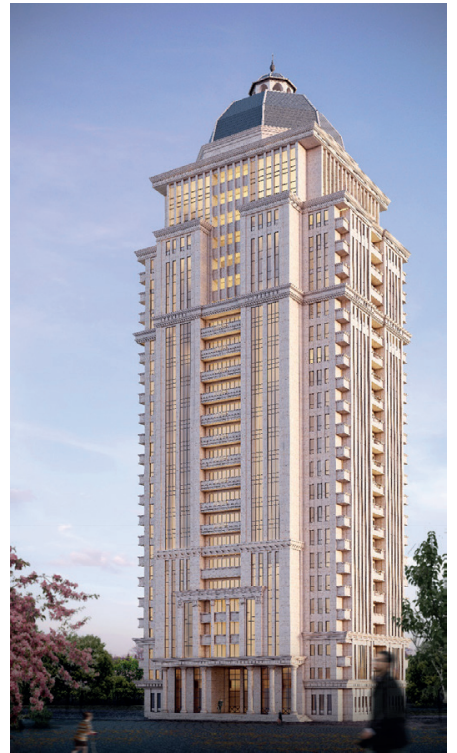

Fig. 3 A rendered snapshot of the Tavrizh project

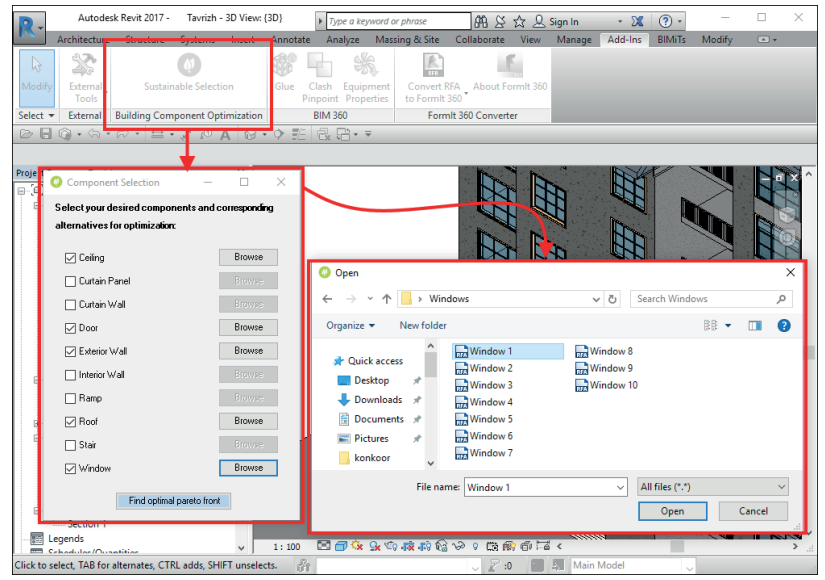

Fig. 4 System interface in the first phase of the framework

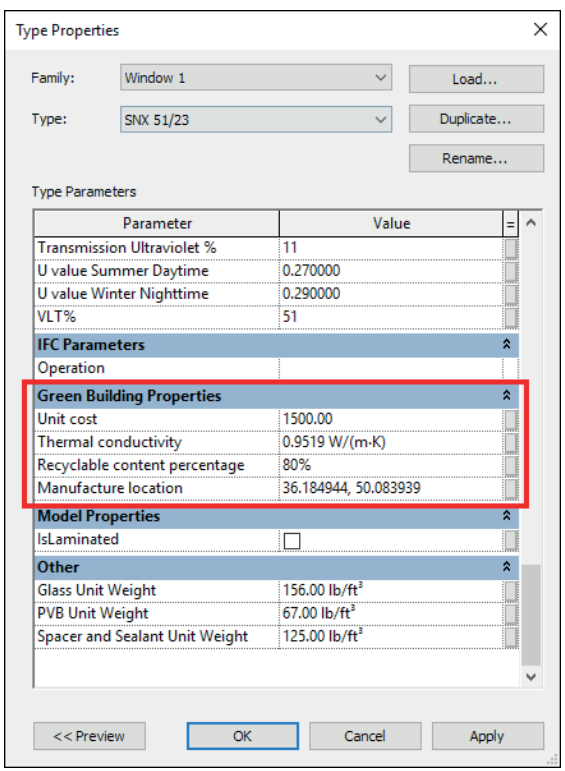

Fig. 5 Properties of a chosen Revit family corresponding alternatives. Selected components are variables of the optimizations phase, and chosen Revit families are considered as their values. So, windows, doors, roofs, exterior walls and ceilings are variables and ten chosen family files for each one are their values. At this stage, initial preparation is terminated. Fig. 4 shows the system interface in the first phase of the framework.

In the next phase, system produce random population and consequently population is updated and optimized by ENSCBO algorithm. All needed data is extracted from the Revit family files by designed plugin in BIM environments. As an example, embedded data of a chosen Revit family are shown in the Fig. 5. The ENSCBO parameters utilized in this paper are as: Population size $=100$, Maximum number of iteration $=1000$, Pro $=0.2$, and Memory size $=5$.

In this case study, four objectives are considered according to purposes of paper. One of the most important objectives is decreasing procurement cost of the components, but it is not a dominated factor in selecting elements. Total cost of the components is computed by Eq. (14).

$T C=\sum_{i=1}^{i=j} Q_{i} \times C_{i}$

Where $C_{i}$ is the unit cost of the component $i, Q_{i}$ is the quantity of that building component and $T C$ is considered as a cost index.

Controlling and lowering the energy consumption of building is another prominent objective. This issue is directly relevant to the environment impact and sustainable development. Therefore, applying materials with more energy saving properties is a priority. In this case, thermal conductivity and heat transfer coefficient are employed and an energy saving estimation is calculated by the following formula:

$E S=\frac{1}{H}=\frac{1}{\sum_{i=1}^{i=j} A_{i} \times K_{i}}$

In this equation, $K_{i}$ is the thermal conductivity of the component $i, A_{i}$ is the total area of that building component and $E S$ is considered as an energy saving index.

To preserve natural resources and achieve a better future, another objective consists of choosing recyclable materials for building components. This causes to further compatibility with nature in the building's life cycle, particularly after demolition. This issue is directly related to "material and resources credit 4: recycled content" of 
Leadership in Energy and Environmental Design (LEED) rating system. Recyclability of utilized components is evaluated by following formula:

$$
R C=\sum_{i=1}^{i=j} W_{i} \times R_{i}
$$

Where $R_{i}$ is recyclable content percentage of the component $i, W_{i}$ is the total weight of that building component and $R C$ is considered as a recyclability index.

Supplying construction materials and building components from nearest manufacturers to construction site leads to less transportation cost and less related $\mathrm{CO}_{2}$ emissions. The main positive impact of this approach is improving the local economy by employing regional human resources and using indigenous materials. Thus the region situation enhancement occurs coincide with construction project progress [5]. This goal observes "material and resources credit 5: regional materials" of LEED rating system. By utilizing latitudes and longitudes of manufactures and project's site, locality of candidates is computed based on Haversine formula as follow:

$$
\begin{aligned}
& a_{i, s}=\sin ^{2}\left(\Delta \varphi_{i, s} / 2\right)+\cos \varphi_{i} \times \cos \varphi_{s} \times \sin ^{2}\left(\Delta \lambda_{i, s} / 2\right) \\
& C_{i, s}=2 \times \arctan 2\left(\sqrt{a_{i, s}}, \sqrt{1-a_{i, s}}\right) \\
& D_{i, s}=R \times C_{i, s} \\
& L C=\sum_{i=1}^{i=j} W_{i} \times D_{i, s}
\end{aligned}
$$

Where $\varphi_{i}$ and $\lambda_{i}$ are longitude and latitude of the $i$ th component manufacture respectively, $\varphi_{s}$ and $\lambda_{s}$ are the longitude and latitude of the project's site; note that angles need to be in radians. $R$ is the earth's radius (mean radius $=6,371 \mathrm{~km}$ ) and $W_{i}$ is the total weight of the $i$ th component. Also LC considered as a localization index.

In summary, considered objectives include reducing cost, increasing energy saving, applying recyclable materials and localization.

$$
\begin{cases}\min & \mathrm{TC} \\ \min & \mathrm{LC} \\ \max & \mathrm{ES} \\ \max & \mathrm{RC}\end{cases}
$$

Output of this phase is an optimal Pareto front, and after sufficient iterations it is presented by the system. In Fig. 6 and Fig. 7, thirty-nine optimal combinations and first combination detail are presented.

Efficiency calculation is carried out for this combinations based on the CCR model. Outputs of the CCR models are recyclability and energy saving index and Inputs of

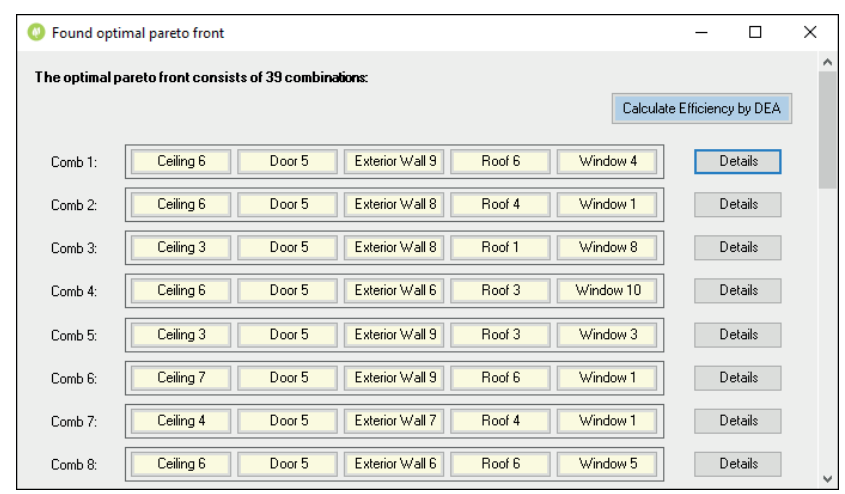

Fig. 6 Found optimal Pareto front by ENSCBO

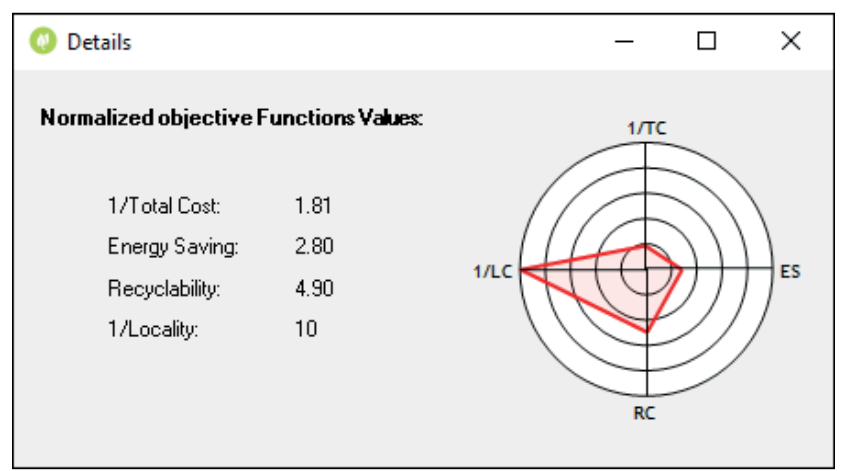

Fig. 7 Normalized objective functions values for Comb 1

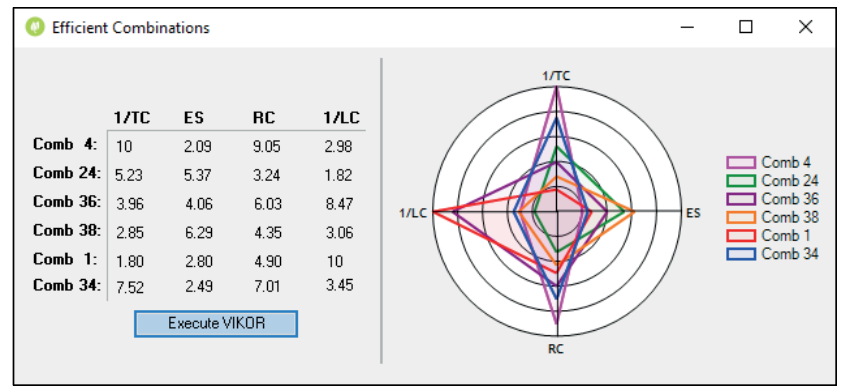

Fig. 8 Efficient combinations calculated by DEA

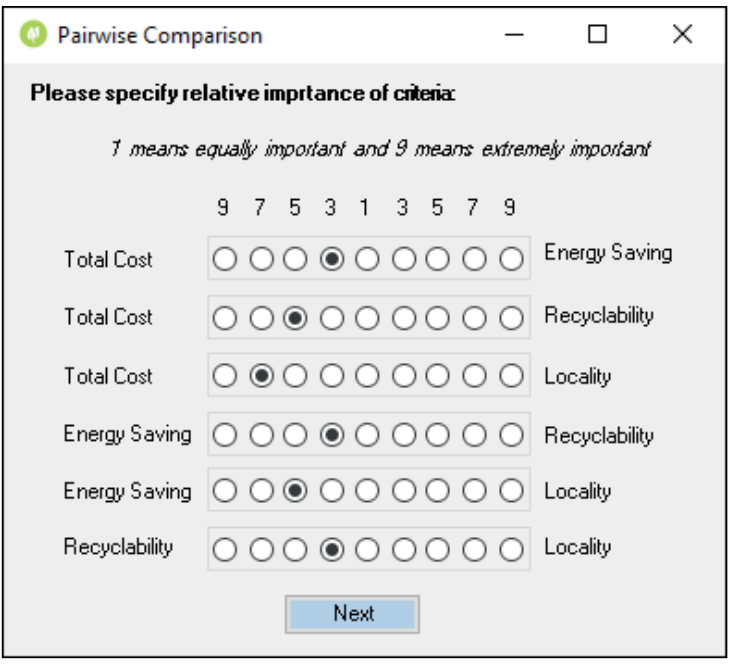

Fig. 9 Pairwise comparison form interface 


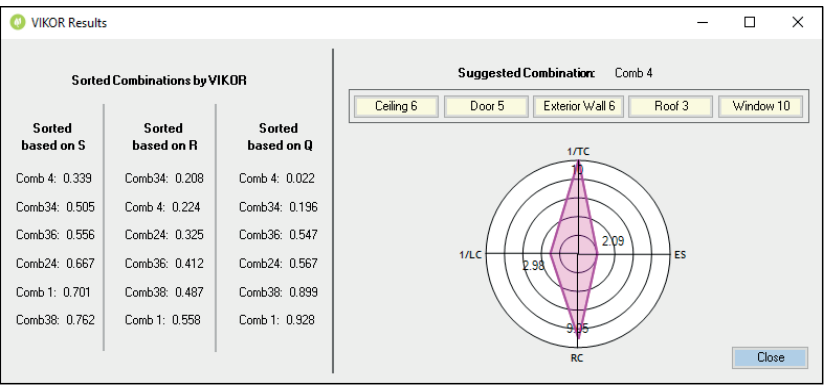

Fig. 10 VIKOR results and its suggested combination

that are cost and localization index. Most efficient combinations with a visual comparison between them, in the form of radar diagram are shown in Fig. 8.

By continuing this process, system asks to do a pairwise comparison between the objectives and to assign a weight to them. The weighting method conforms the Saaty scale in the range of 1 to 9. In Fig. 9, plugin comparing form and the assigned importance are depicted.

According to these weights and VIKOR procedure, final combination will be selected. VIKOR result and the suggested combination are presented in Fig. 10.

The suggested combination for the project is achieved by performing various phases from $10^{5}$ possible combinations.

\section{Conclusions}

This paper has outlined a framework for choosing the best building components considering sustainability aspects in a BIM environment. Reducing cost, increasing energy saving, applying recyclable materials and localization are objective functions of this approach. In the path of choosing the best combination, several phases such as optimization phase are devised. In this phase, with the help of a meta-heuristic algorithm (e.g. ENSCBO), non-dominated combinations are found and entered to the subsequent phases. In the next phase, efficiency of the qualified combinations is evaluated by the DEA, and then the compromise ranking method, VIKOR, ranks the most efficient ones and determines the compromise solution according to the user's preferences in the last phase.

In order to accomplish this framework on construction projects a plugin is developed in Revit Autodesk and its performance is illustrated through a case study. The main material of this plugin is pre-defined rich Revit family files for each considered building component as their alternatives. Consequently, this approach provides a valuable tool for the project stakeholder in selecting the best combinations among the available alternatives.

\section{Acknowledgement}

This research is supported by Iransazeh company. The authors would like to thank the company managers for their support in providing research requirements.

\section{References}

[1] Keeble, B. R. "The Brundtland report: 'Our common future' ", Medicine and War, 4(1), pp. 17-25, 1988. https://doi.org/10.1080/07488008808408783

[2] Rao, R. V. "A decision making methodology for material selection using an improved compromise ranking method", Materials \& Design, 29(10), pp. 1949-1954, 2008. https://doi.org/10.1016/j.matdes.2008.04.019

[3] Flórez, L., Castro-Lacouture, D., Irizarry, J. "Impact of sustainability perceptions on the purchasability of materials in construction projects", In: Construction Research Congress 2010: Innovation for Reshaping Construction Practice, Banff, Alberta, Canada, 2010, pp. 1447-1456. https://doi.org/10.1061/41109(373)145

[4] Ashby, M. F. "Multi-objective optimization in material design and selection", Acta Materialia, 48(1), pp. 359-369, 2000. https://doi.org/10.1016/S1359-6454(99)00304-3

[5] Ogunkah, I., Yang, J. "Investigating Factors Affecting Material Selection: The Impacts on Green Vernacular Building Materials in the Design-Decision Making Process", Buildings, 2(1), pp. 1-32, 2012 .

https://doi.org/10.3390/buildings2010001

[6] Akadiri, P. O., Olomolaiye, P. O., Chinyio, E. A. "Multi-criteria evaluation model for the selection of sustainable materials for building projects", Automation in Construction, 30, pp. 113-125, 2013. https://doi.org/10.1016/j.autcon.2012.10.004

[7] Fu, C., Aouad, G., Lee, A., Mashall-Ponting, A., Wu, S. "IFC model viewer to support nD model application", Automation in Construction, 15(2), pp. 178-185, 2006.

https://doi.org/10.1016/j.autcon.2005.04.002

[8] Chan, J. W. K., Tong, T. K. L. "Multi-criteria material selections and end-of-life product strategy: Grey relational analysis approach", Materials and Design, 28(5), pp. 1539-1546, 2007. https://doi.org/10.1016/j.matdes.2006.02.016

[9] Wu, W.-Y., Sukoco, B. M., Li, C.-Y., Chen, S. H. "An integrated multi-objective decision-making process for supplier selection with bundling problem", Expert Systems with Applications, 36(2), pp. 2327-2337, 2009. https://doi.org/10.1016/j.eswa.2007.12.022

[10] Zhou, C.-C., Yin, G.-F., Hu, X.-B. "Multi-objective optimization of material selection for sustainable products: Artificial neural networks and genetic algorithm approach", Materials \& Design, 30(4), pp. 1209-1215. 2009. https://doi.org/10.1016/j.matdes.2008.06.006

[11] Castro-Lacouture, D., Sefair, J. A., Flórez, L., Medaglia, A. L. "Optimization model for the selection of materials using a LEEDbased green building rating system in Colombia", Building and Environment, 44(6), pp. 1162-1170, 2009. https://doi.org/10.1016/j.buildenv.2008.08.009 
[12] Hamdy, M., Hasan, A., Siren, K. "Applying a multi-objective optimization approach for Design of low-emission cost-effective dwellings", Building and Environment, 46(1), pp. 109-123, 2011. https://doi.org/10.1016/j.buildenv.2010.07.006

[13] Florez, L., Castro-Lacouture, D. "Optimization model for sustainable materials selection using objective and subjective factors", Materials and Design, 46, pp. 310-321, 2013. https://doi.org/10.1016/j.matdes.2012.10.013

[14] Marzouk, M., Metawie, M., Hisham, M., Al-Sulahi, I., Kamal, M., Al-Gahtani, K. "Modeling Sustainable Building Materials in Saudi Arabia", In: 2014 International Conference on Computing in Civil and Building Engineering, Orlando, Florida, United States, 2014, pp. 1546-1553.

https://doi.org/10.1061/9780784413616.192

[15] Jalaei, F., Jrade, A., Nassiri, M. "Integrating decision support system (DSS) and building information modeling (BIM) to optimize the selection of sustainable building components", Journal of Information Technology in Construction, 20, pp. 399-420, 2015. Available at https://www.itcon.org/paper/2015/25 [Accessed: 06.11.2018 ]

[16] Liu, S., Meng, X., Tam, C. "Building information modeling based building design optimization for sustainability", Energy and Buildings, 105, pp. 139-153, 2015. https://doi.org/10.1016/j.enbuild.2015.06.037

[17] Akanmu, A., Asfari, B., Olatunji, O. "BIM-Based Decision Support System for Material Selection Based on Supplier Rating", Buildings, 5(4), pp. 1321-1345, 2015. https://doi.org/10.3390/buildings5041321

[18] Govindan, K., Shankar, K. M., Kannan, D. "Sustainable material selection for construction industry - A hybrid multi criteria decision making approach", Renewable and Sustainable Energy Reviews, 55, pp. 1274-1288, 2016. https://doi.org/10.1016/j.rser.2015.07.100

[19] Dawood, M. H. "BIM based optimal life cycle cost of sustainable house framework", In: 2016 3rd MEC International Conference on Big Data and Smart City, Muscat, Oman, 2016, pp. 279-283. https://doi.org/10.1109/ICBDSC.2016.7460381

[20] Ahmad, T., Thaheem, M. J. "Economic sustainability assessment of residential buildings: A dedicated assessment framework and implications for BIM", Sustainable Cities and Society, 38, pp. 476-491, 2018. https://doi.org/10.1016/j.scs.2018.01.035

[21] Kaveh, A. "Advances in Metaheuristic Algorithms for Optimal Design of Structures", 2nd ed., Springer, Cham, Switzerland, 2017. https://doi.org/10.1007/978-3-319-46173-1

[22] Csébfalvi, A. "A hybrid meta-heuristic method for continuous engineering optimization", Periodica Polytechnica-Civil Engineering, 53(2), pp. 93-100, 2009.

https://doi.org/10.3311/pp.ci.2009-2.05

[23] Danka, S., Csébfalvi, A. "A Hybrid Metaheuristic for Project Scheduling Problems with Fuzzy Activity Durations to support the Ö s-Dráva Water Management Programme", In: Proceedings of the Eighth International Conference on Engineering Computational Technology, Dubrovnik, Croatia, 2012, pp. 1-20. Available at http://webapp.tudelft.nl/proceedings/ect2012/pdf/danka.pdf [Accessed: 06.11.2018]
[24] Csébfalvi, A., "Hybrid Metaheuristic Methods in Truss Opti-mization: A Review", Computational Technology Reviews, 8, pp. 63-92, 2013. https://doi.org/10.4203/ctr.8.3

[25] Csébfalvi, A., Csébfalvi, G. "Fair Comparison of Population-based Heuristic Approaches", In: Proceedings of the 4th International Joint Conference on Computational Intelligence, Barcelona, Spain, 2012, pp. 206-309. https://doi.org/10.5220/0004168403060309

[26] Kaveh, A., Mahdavi, V. R. "Colliding bodies optimization: A novel meta-heuristic method", Computers \& Structures, 139, pp. 18-27, 2014. https://doi.org/10.1016/j.compstruc.2014.04.005

[27] Kaveh, A., Moghaddam, M. R., Khanzadi, M. "Efficient multiobjective optimization algorithms for construction site layout problem", Scientia Iranica, 25(4), pp. 2051-2062, 2018. https://doi.org/10.24200/SCI.2017.4216

[28] Kaveh, A., Khanzadi, M., Alipour, M., Moghaddam, M. R. "Construction Site Layout Planning Problem Using Two New Metaheuristic Algorithms", Iranian Journal of Science and Technology, Transactions of Civil Engineering, 40(4), pp. 263-275, 2016. https://doi.org/10.1007/s40996-016-0041-0

[29] Kaveh, A., Moghaddam, M. R. "A hybrid WOA-CBO algorithm for construction site layout planning problem", Scientia Iranica, 25(3), pp. 1094-1104, 2018. https://doi.org/10.24200/sci.2017.4212

[30] Kaveh, A., Ilchi Ghazaan, M. "Enhanced colliding bodies optimization for design problems with continuous and discrete variables", Advances in Engineering Software, 77, pp. 66-75, 2014. https://doi.org/10.1016/j.advengsoft.2014.08.003

[31] Deb, K., Pratap, A., Agarwal, S., Meyarivan, T. "A fast and elitist multiobjective genetic algorithm: NSGA-II", IEEE Transactions on Evolutionary Computation, 6(2), pp. 182-197, 2002. https://doi.org/10.1109/4235.996017

[32] Ehrgott, M., Holder, A., Nohadani, O. "Uncertain Data Envelopment Analysis", European Journal of Operational Research, 268(1), pp. 231-242, 2018. https://doi.org/10.1016/j.ejor.2018.01.005

[33] Charnes, A., Cooper, W. W., Rhodes, E. "Measuring the efficiency of decision making units", European Journal of Operational Research, 2(6), pp. 429-444, 1978. https://doi.org/10.1016/0377-2217(78)90138-8

[34] Tzeng, G.-H., Huang, J.-J. "Multiple Attribute Decision Making", 1st ed., Chapman and Hall/CRC, New York, U.S.A., 2011. https://doi.org/10.1201/b11032 